\title{
Associations between obesity candidate gene polymorphisms (fat mass and obesity-associated (FTO), melanocortin-4 receptor (MC4R), leptin (LEP) and leptin receptor (LEPR)) and dietary intake in pregnant women
}

\author{
Maisa C. Martins ${ }^{1,2}$, Janet Trujillo ${ }^{1}$, Ana Amélia Freitas-Vilela ${ }^{1,3}$, Dayana R. Farias ${ }^{1,2}$, Eliane L. Rosado ${ }^{4}$, \\ Cláudio J. Struchiner ${ }^{5}$ and Gilberto $\mathrm{Kac}^{1 *}$ \\ ${ }^{1}$ Nutritional Epidemiology Observatory, Department of Social and Applied Nutrition, Institute of Nutrition Josué de Castro, \\ Federal University of Rio de Janeiro, Rio de Janeiro, 21941-902, Brazil \\ ${ }^{2}$ Graduate Program in Nutrition, Institute of Nutrition Josué de Castro, Federal University of Rio de Janeiro, \\ Rio de Janeiro, 21941-902, Brazil \\ ${ }^{3}$ Special Academic Unit of Health Sciences, Goiás Federal University, Jataí-Goiás, 75803-100, Brazil \\ ${ }^{4}$ Department of Nutrition and Dietetics, Institute of Nutrition Josué de Castro, Federal University of Rio de Janeiro, \\ Rio de Janeiro, 21941-902, Brazil \\ ${ }^{5}$ Department of Endemic Diseases, National School of Public Health, Oswaldo Cruz Foundation, Rio de Janeiro, 21041-210, Brazil \\ (Final revision received 30 July 2017 - Submitted 20 April 2018 - Accepted 26 April 2018 - First published online 12 June 2018 )
}

\section{Abstract}

Genetic variants associated with dietary intake may be important as factors underlying the development of obesity. We investigated the associations between the obesity candidate genes (fat mass and obesity-associated (FTO), melanocortin- 4 receptor $(M C 4 R)$, leptin $(L E P)$ and leptin receptor) and total energy intake and percentage of energy from macronutrients and ultra-processed foods before and during pregnancy. A sample of 149 pregnant women was followed up in a prospective cohort in Rio de Janeiro, Brazil. A FFQ was administered at 5-13 and 30-36 weeks of gestation. Genotyping was performed using real-time PCR. Associations between polymorphisms and the outcomes were investigated through multiple linear regression and ANCOVA having pre-pregnancy dietary intake as a covariate. The A-allele of FTOrs 9939609 was associated with a $-6.5 \%(95 \% \mathrm{CI}-12 \cdot 3,-0.4)$ decrease in the percentage of energy from protein and positively associated with the percentage of energy from carbohydrates before pregnancy $(\beta=2 \cdot 6 ; 95 \%$ CI $0 \cdot 5,4 \cdot 8)$ and with a $13.3 \%(95 \%$ CI $0 \cdot 7,27 \cdot 5)$ increase in the total energy intake during pregnancy. The C-allele of $M C 4 R-r s 17782313$ was associated with a $-7 \cdot 6 \%(95 \% \mathrm{CI}-13 \cdot 8,-1 \cdot 0)$ decrease in the percentage of energy from protein, and positively associated with the percentage of energy from ultra-processed foods ( $\beta=5 \cdot 4 ; 95 \% \mathrm{CI} 1 \cdot 1$, 9.8) during pregnancy. ANCOVA results revealed changes in dietary intake from pre-pregnancy to pregnancy for FTO-rs 9939609 (percentage of energy from ultra-processed foods, $P=0 \cdot 03$ ), MC4R-rs17782313 (total energy intake, $P=0.02$ ) and LEP-rs 7799039 (total energy intake, $P=0.04$; percentage of energy from protein, $P=0.04$ ). These findings suggest significant associations between FTO-rs9939609, MC4Rrs17782313 and LEP-rs7799039 genes and the components of dietary intake in pregnant women.

Key words: Dietary intake: Pregnant women: Polymorphisms: Energy intake

There is general agreement that the current obesity epidemic and diet-related non-communicable diseases (NCD) are largely associated with an environment that promotes excessive food intake and discourages physical activity in multiple ways ${ }^{(1,2)}$. Globally, the consumption of healthy foods has increased modestly during the past two decades. In contrast, the intake of unhealthy foods has increased to a greater extent ${ }^{(3)}$. Although environmental factors have an important role in the development of obesity, genetic factors also make a significant contribution in determining an individual's susceptibility to obesity $^{(4)}$ through a full series of potential mechanisms governing pathways and regulatory systems at different levels, including intake and energy expenditure ${ }^{(5)}$.

Studies of candidate genes for obesity susceptibility have allowed the identification of important genes and SNP involved in the mechanisms of dietary consumption, such as energy intake, food preferences and satiety responsiveness ${ }^{(6-8)}$. The $r 9939609$ SNP of the fat mass and obesity-associated $(F T O)$ gene, along with the $r s 7799039 \mathrm{SNP}$ of the leptin (LEP) gene and the $r 1137101$ $\mathrm{SNP}$ of the leptin receptor ( $L E P R)$ gene, has been associated with

Abbreviations: $F T O$, fat mass and obesity-associated; GW, gestational weeks; $L E P$, leptin; $L E P R$, leptin receptor; $M C 4 R$, melanocortin- 4 receptor. 
total energy intake ${ }^{(9,10)}$. Further, the $r s 17782313$ SNP of the melanocortin-4 receptor $(M C 4 R)$ gene has been associated with food intake and eating behaviour patterns ${ }^{(11,12)}$.

Whereas total energy intake is a vital aspect of food intake, the macronutrient composition of food or dietary patterns may be equally important as factors underlying the development of obesity $^{(13)}$. Consumption of ultra-processed foods has become a common practice worldwide ${ }^{(14,15)}$. Ultra-processed foods are defined as industrial formulations, typically with five or more ingredients that, besides salt, sugar, oils and fats, include additives that are used to give the products desirable sensory properties $^{(16,17)}$. This type of food is associated with less healthy diets and strongly implicated in over-consumption, weight gain, obesity and chronic health problems ${ }^{(14)}$.

Nutritional demands increase during pregnancy owing to the metabolic and physiological changes ${ }^{(18)}$, but the polymorphism may also be associated with dietary preferences over time affecting food intake and, consequently, weight gain. Maternal obesity and excessive gestational weight gain are major risk factors for gestational diabetes, pre-eclampsia and fetal adiposity ${ }^{(19,20)}$. Consequently, obesity in pregnancy is placing a considerable burden on healthcare services and resources ${ }^{(21)}$. To the best of our knowledge, there are no studies in the literature that have evaluated the association between these SNP (FTO, MC4R, LEP and $L E P R)$ and dietary intake during pregnancy.

Given the potential importance of weight management interventions in pregnancy and owing to the association between dietary intake and weight gain and regulation, this study may help shed light on this important topic and provide insights to guide the advances of maternal nutrition during pregnancy. In this way, the aims of this study were: (1) to investigate whether obesity candidate gene (FTO-rs9939609; MC4R-rs17782313; LEP-rs7799039 and LEPR-rs1137101) polymorphisms are associated with daily total energy intake and percentage of energy from macronutrients and ultraprocessed foods in both time points (pre-pregnancy and pregnancy), and (2) to test whether these genes are associated with dietary intake changes from pre-pregnancy to pregnancy. We hypothesised that variation in FTO, MC4R, LEP and LEPR gene SNP may be associated with an increased total energy intake, as well as increased energy from carbohydrates, fats and ultraprocessed foods, and with decreased energy from proteins before and during pregnancy.

\section{Methods}

\section{Study design and subjects}

This study comprised a prospective cohort of pregnant women attending a prenatal care service offered by a public health centre in Rio de Janeiro, Brazil. The enrolment of women occurred from November 2009 to October 2011, and the follow-up lasted until July 2012. The Rio de Janeiro Municipal Health Secretary Research Ethics Committee approved the study procedures (reference no. 0139.0.314.000-09). All participants signed a term of consent freely and spontaneously, detailing all procedures to be carried out, according to Brazilian Resolution $466 / 2012$
Women who met the following eligibility criteria were invited to participate in the study: had $<13$ weeks of gestation at baseline, were 20-40 years of age, had no history of infectious diseases or NCD (except obesity) and intended to attend prenatal care in the selected health centre. The follow-up times occurred between 5 and 13 (baseline), 20 and 26 and 30 and 36 gestational weeks (GW).

A total of 322 pregnant women were invited, and 299 (92.7\%) agreed to participate in the study. After recruitment, seventynine women were excluded. After exclusions, the baseline total sample comprised 220 pregnant women. From the baseline to the third visit (30-36GW), nine follow-up losses occurred. Furthermore, we excluded women who did not have blood samples for genotyping ( $n$ 62). The final sample of this study was composed of 149 women with genotyping (FTO, $n$ 146; $M C 4 R, n$ 145; LEP, $n 147$ and LEPR, $n$ 147) (Fig. 1).

\section{Dietary intake assessments}

The dietary intake was evaluated using a semi-quantitative FFQ based on a version originally developed and validated for the adult population of Rio de Janeiro ${ }^{(22)}$. The participants were asked to report the frequency of consumption of the food items contained in the FFQ over the 6 months before the interview. The dietary intake data collected at $5-13 \mathrm{GW}$ covered the prepregnancy period and the data collected at 30-36 GW refer to dietary intake of women during pregnancy. To capture any other relevant food, we included a question at the end of the FFQ: 'Do you regularly eat other foods that have not been asked?' Very few women reported eating other food items not included on the FFQ, and this information was not included in the analysis. The FFQ was composed of eighty-two food items with eight frequency options that were transformed into daily frequency, as follows: $>3$ times $/ \mathrm{d}=4,2-3$ times $/ \mathrm{d}=2 \cdot 5,1$ time/ $\mathrm{d}=1$, 5-6 times/week $=0 \cdot 79,2-4$ times/week $=0 \cdot 43,1$ time/ week $=0.14,1-3$ times $/$ month $=0.07$ and never or hardly ever $=0$. Portion sizes were converted into grams or millilitres, based on the Brazilian household measures table ${ }^{(23)}$. The daily nutrient amount for a given food was calculated by multiplying the usual portion size per daily frequency and its nutrient content based on data from the Brazilian Table of Food Composition $^{(24)}$, and we added food items from the US Department of Agriculture National Nutrient Database for Standard ${ }^{(25)}$.

Macronutrients and alcohol consumptions (g/d) were calculated by summing the data for the respective macronutrient/ alcohol across all food items. These values were then converted into energy by multiplying the total grams of the macronutrient/ alcohol by the number of energy content per gram.

Ultra-processed foods were classified in accordance with the official Brazilian national food and nutrition guideline ${ }^{(16)}$ and with the new food classification system (NOVA) ${ }^{(17)}$, which considers the extent and the purpose of industrial food processing. Ultra-processed foods were represented as follows: breads; cakes, cookies/crackers or stuffed cookies and sweet or savoury packaged snacks; dairy products (cheese and yogurt); margarine and mayonnaise; pasta and noodles; processed meats (burgers, sausages/frankfurters, cold cuts: bologna, ham, salami); candies (ice cream, candies/caramels, chocolate 


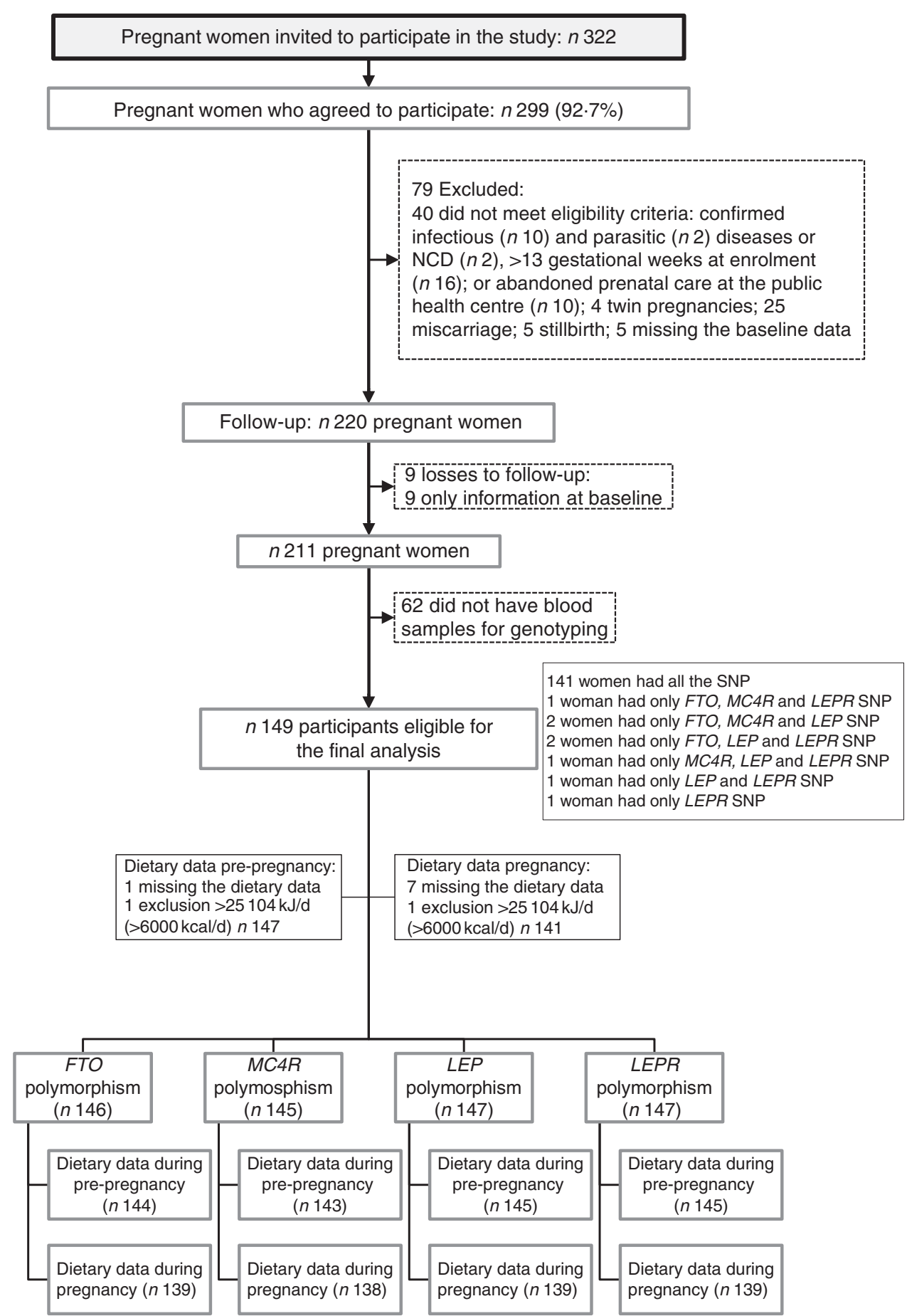

Fig. 1. Flow chart illustrating the recruitment and selection of the study sample. NCD, non-communicable diseases; FTO, fat mass and obesity-associated gene; $M C 4 R$, melanocortin-4 receptor gene; $L E P$, leptin; $L E P R$, leptin receptor.

powder, chocolate bars/bonbons, sweet dairy); French fries, chips or shoestring potatoes, fried/baked salted pastries and pizza dishes; and soft drinks and alcoholic beverages (vodka).

\section{DNA isolation and SNP genotyping}

Non-fasting venous blood samples $(5 \mathrm{ml})$ were collected at the baseline, processed and stored at $-80^{\circ} \mathrm{C}$ until polymorphism analyses. DNA was isolated from whole blood samples by the proteinase $\mathrm{K}$ and phenol-chloroform technique. Genotyping was performed using real-time PCR amplification method (StepOnePlus; Life Technologies) with an allelic discrimination assay (TaqMan ${ }^{\circledR}$ Genotyping Master Mix assay; Life Technologies). The accuracy of genotyping was evaluated by performing a duplicate analysis of $10 \%$ of the sample with $\geq 99 \%$ agreement rates. 


\section{Covariate assessment}

A standardised questionnaire was administered at baseline (5-13 GW) to obtain the following maternal variables: age (years), education (years of schooling), per-capita family income (Real-R\$), leisure-time physical activity practice before pregnancy (yes/no), smoking habits (non-smoking, former smoker and current smoker), alcohol consumption (no/yes), parity (number of deliveries) and self-reported skin colour (white/black/mixed).

Pre-pregnancy BMI was calculated using the self-reported pre-pregnancy weight at baseline and height was measured with a portable stadiometer (Seca Ltd) until 13 weeks of gestation according to standardised procedures ${ }^{(26)}$.

The gestational age was estimated based on the first ultrasound performed before 24 weeks of gestation ${ }^{(27)}$; however, the reported date of the last menstrual period was used if the ultrasound data were not available ( $n$ 2).

\section{Statistical analyses}

The maternal characteristics were described as a numbers and percentages or medians and interquartile ranges (IQR, 25th75 th percentiles) and mean values and $95 \%$ CI. The normality of distributions was tested using the Shapiro-Wilk test. Data that were not normally distributed (energy intake and percentage of energy from protein) were transformed to the natural logarithmic $(\ln )$ scale $\left(\ln (x)=\log _{e}(x)\right.$ ). After log-transformation, the QQ plots and Shapiro-Wilk test did not provide evidence that the data did not follow a normal distribution. Log-transformed values were used before data analysis, but estimates provided in the results section were back-transformed using the inverse of the logarithmic function $\left(e^{\ln (x)}=x\right)$, and data are presented as geometric means and $95 \%$ CI. Comparisons between genotypes and outcomes were performed using Student's $t$ test. Because the number of minor allele homozygotes for all SNP was small in our sample, only the dominant genetic model was tested instead of the additive and recessive genetic models.

Adjusted linear regression models were performed to assess cross-sectional associations between SNP and the outcomes. Characteristics related to the mother and (log) total energy intake with $P<0.20$ in univariate analysis were included in the adjusted models. The following variables reached this condition and were included in the adjusted models: maternal age, selfreported skin colour, parity, gestational age and per-capita income. Analyses of the percentage of energy from macronutrients and ultra-processed foods were also adjusted for total energy intake. The results for log-transformed variables (total energy intake and percentage of energy from protein) were presented in \% change with $95 \%$ CI to make interpretation more straightforward. The assumption of normal distribution of the residuals was examined using QQ plots.

ANCOVA models were fitted to explore the associations between SNP and dietary intake changes during pregnancy. In these analyses, the dietary intake value during pregnancy was entered as the dependent variable. We contrasted two groups under the dominant genetic model. Group 1 comprised homozygotes for the minor allele and heterozygotes carrying the same allele. This latter group was then contrasted to the wild-type group (homozygote major allele). The variable coded according to the previous definition was entered as an explanatory factor in the ANCOVA model. Its effect was adjusted for the remaining covariates, such as maternal age at baseline, selfreported skin colour, parity, gestational age at baseline and respective dietary intake value at baseline (pre-pregnancy). Correlations between outcomes during pregnancy and possible covariates were analysed using Pearson's correlation test. The assumption that covariates were independent of genotypes was tested using Student's $t$ test. Homogeneity of genotypic effects according to pre-pregnancy dietary intake was tested using an interaction term between the genotype groups and prepregnancy dietary intake in the corresponding ANCOVA model. When there was no interaction, a full factorial model was chosen. The model fit was assessed graphically using residual plots. The results are presented as adjusted means and $95 \% \mathrm{CI}$.

We estimated the needed sample size for the following Cohen's effects sizes: small (0.2), medium (0.5) and large (0.8), based on a power of $80 \%$ and a CI level of $5 \%$ (considering $t$ tests of means). The effect size was considered as the standardised difference between the two groups of risk allele carriers and non-risk allele homozygotes in pre-pregnancy measurements. The mean differences between the groups of the A-carriers and $\mathrm{G}$ homozygote of the polymorphism LEPrs 7799039 would be $678 \mathrm{~kJ} / \mathrm{d}(162 \mathrm{kcal} / \mathrm{d})$ (small effect), $1694.5 \mathrm{~kJ} / \mathrm{d}$ ( $405 \mathrm{kcal} / \mathrm{d}$ ) (medium effect) and $2707 \mathrm{~kJ} / \mathrm{d}$ $(647 \mathrm{kcal} / \mathrm{d})$ (large effect) for pre-pregnancy total energy $(\mathrm{kJ} / \mathrm{d})$ ( $\mathrm{kcal} / \mathrm{d})$. It resulted in total sample sizes of 794 (small effect), 130 (medium effect) and 52 (large effect) pregnant women (taking into account a group ratio of $1: 2$ as in polymorphism LEP-rs 7799039). These analyses were developed using the 'pwr' package of the R-project statistical software (version 1.0.143) $)^{(28)}$ and G*Power software (version 3.1.9.2) ${ }^{(29)}$. We calculated the power implied by our sample size when comparing each outcome and polymorphism. Power calculations were performed using the 'GeneticsDesign' package of R-project statistical software (version 1.0.143) ${ }^{(28)}$ under the dominant model - that is, contrasting the homozygote minor allele + heterozygotes carriers to the wild-type group (homozygote major allele). Our choice of the remaining input parameters in this function preserved the same logic. More specifically, options 'delta' (difference in means of dietary intake at baseline relative to the two groups being compared) and 'freq' (allele frequency of 'disease' allele in our sample) reflect the same coding of the main analysis. In this exercise, $\alpha$ was set to 0.05 and the standard deviations estimated from the target outcome at baseline (online Supplementary Table S4).

All of the statistical analyses were conducted using STATA (version 12.0; StataCorp) software, and the significance level was defined as $5 \%$. Corrections for multiple testing were not performed because they may not be necessarily required in exploratory studies, such as the present one. Considering that the likelihood of type II errors is increased by corrections for multiple testing, such as Bonferroni adjustments, and considering that truly important differences may not be deemed significant ${ }^{(30)}$, such adjustments were not performed in the present study. 


\section{Results}

\section{Characteristics of the study sample}

The average age of the 149 women was 27 years old $(\mathrm{IQR}=$ $22-31$ ), and they had completed $9 \cdot 0$ (IQR $=7-11$ ) years of education. The frequency of women with a pre-pregnancy BMI $\geq 25 \mathrm{~kg} / \mathrm{m}^{2}$ was $34.9 \%$ (Table 1 ). The difference in total energy intake (geometric mean) before and during pregnancy was not significant $(P=0 \cdot 86)$ (data not shown).

Table 1. Maternal characteristics at the baseline and pre-pregnancy and pregnancy dietary intake

(Medians and interquartile ranges (IQR); numbers and percentages; geometric means and $95 \%$ confidence intervals)

\begin{tabular}{|c|c|c|c|c|}
\hline \multirow[b]{2}{*}{ Maternal characteristics } & \multicolumn{4}{|c|}{ Value study sample } \\
\hline & Median & IQR & $n$ & $\%$ \\
\hline \multicolumn{5}{|l|}{$\begin{array}{l}\text { Socio-demographic and lifestyle } \\
(n \text { 149) }\end{array}$} \\
\hline Age (years) & 27 & $22-31$ & & \\
\hline Education (years) & 9 & $7-11$ & & \\
\hline Per-capita family income $(\mathrm{R} \$)^{\star}$ & $475 \cdot 0$ & $315 \cdot 0-667 \cdot 0$ & & \\
\hline \multicolumn{5}{|l|}{ Parity (number of parturitions) } \\
\hline 0 & & & 57 & $38 \cdot 3$ \\
\hline$\geq 1$ & & & 92 & 61.7 \\
\hline \multicolumn{5}{|l|}{$\begin{array}{l}\text { Early-pregnancy alcohol } \\
\text { consumption }\end{array}$} \\
\hline No & & & 121 & $81 \cdot 2$ \\
\hline Yes & & & 28 & 18.8 \\
\hline \multicolumn{5}{|l|}{$\begin{array}{l}\text { Early-pregnancy smoking } \\
\text { habit }\end{array}$} \\
\hline Non-smoking & & & 108 & 72.5 \\
\hline Former smoker & & & 31 & 20.8 \\
\hline Current smoker & & & 10 & 6.7 \\
\hline \multicolumn{5}{|l|}{ LTPA before pregnancy* ${ }^{*}$} \\
\hline No & & & 119 & $81 \cdot 0$ \\
\hline Yes & & & 28 & $19 \cdot 0$ \\
\hline \multicolumn{5}{|l|}{ Self-reported skin colour } \\
\hline White & & & 39 & $26 \cdot 2$ \\
\hline Black & & & 35 & 23.5 \\
\hline Brown (mixed race) & & & 75 & $50 \cdot 3$ \\
\hline \multicolumn{5}{|l|}{$\begin{array}{l}\text { Pre-pregnancy BMI }\left(\mathrm{kg} / \mathrm{m}^{2}\right) \\
\text { status }\end{array}$} \\
\hline Underweight $(<18.5)$ & & & 5 & 3.4 \\
\hline Normal weight (18.5-24.9) & & & 92 & 61.7 \\
\hline Overweight (25.0-29.9) & & & 33 & $22 \cdot 2$ \\
\hline Obesity $(\geq 30.0)$ & & & 19 & $12 \cdot 7$ \\
\hline \multicolumn{3}{|l|}{$\begin{array}{l}\text { Pre-pregnancy daily dietary } \\
\text { intake }(n 147) \dagger\end{array}$} & Mean & $95 \% \mathrm{Cl}$ \\
\hline \multirow{2}{*}{\multicolumn{3}{|c|}{$\begin{array}{l}\text { Total energy }(\mathrm{kJ} / \mathrm{d}) \S \\
\text { Total energy }(\mathrm{kcal} / \mathrm{d}) \S\end{array}$}} & 9489 & 8991,10021 \\
\hline & & & 2268 & 2149,2395 \\
\hline \multicolumn{5}{|l|}{$\%$ of energy from } \\
\hline \multicolumn{3}{|l|}{ Protein§ } & 15.9 & $15 \cdot 4,16 \cdot 4$ \\
\hline \multicolumn{3}{|l|}{$\begin{array}{l}\text { Carbohydrates } \\
\text { Fat }\end{array}$} & $56 \cdot 2$ & $55 \cdot 2,57 \cdot 2$ \\
\hline \multirow{2}{*}{\multicolumn{3}{|c|}{ Ultra-processed foods }} & $26 \cdot 7$ & $25 \cdot 9,27 \cdot 5$ \\
\hline & & & 47.4 & $45 \cdot 4,49 \cdot 5$ \\
\hline \multicolumn{5}{|l|}{$\begin{array}{l}\text { Pregnancy daily dietary intake } \\
(n \text { 141) } \pm\end{array}$} \\
\hline Total energy $(\mathrm{kJ} / \mathrm{d}) \S$ & & & 9556 & 9054,10083 \\
\hline Total energy $(\mathrm{kcal} / \mathrm{d}) \S$ & & & 2284 & 2164,2410 \\
\hline \multicolumn{5}{|l|}{$\%$ of energy from } \\
\hline Protein§ & & & $16 \cdot 0$ & $15 \cdot 5,16 \cdot 6$ \\
\hline Carbohydrates & & & 58.3 & $57.4,59.2$ \\
\hline Fat & & & $25 \cdot 2$ & $24 \cdot 4,25 \cdot 9$ \\
\hline Ultra-processed foods & & & $44 \cdot 2$ & $41 \cdot 9,46 \cdot 4$ \\
\hline
\end{tabular}

$\mathrm{R} \$$, real; LTPA, leisure-time physical activity.

* Variables with missing information: three missing values for per-capita family income and two missing values for LTPA.

† One missing value for FFQ and one exclusion because implausible total energy intake (>25104 kJ/d (>6000 kcal/d)).

₹ Seven missing values for FFQ and one exclusion because implausible total energy intake $((>25104 \mathrm{~kJ} / \mathrm{d}(>6000 \mathrm{kcal} / \mathrm{d}))$.

$\S$ Natural logarithms values were used for variables without normal distribution, but estimates provided in the table were back-transformed.

\section{Genotype frequencies}

The minor allele frequencies of FTO-rs9939609, MC4Rrs17782313, LEP-rs 7799039 and LEPR-rs1137101 were $42 \cdot 0 \%$ (A-allele), $19.0 \%$ (C-allele), $31.0 \%$ (A-allele) and $50.0 \%$ (G-allele), respectively. The genotype distributions were in accordance with the Hardy-Weinberg equilibrium $(P>0.05)$ (online Supplementary Table S1).

\section{Daily total energy intake}

Carriers of the A-allele of FTO-rs9939609 gene presented a higher geometric mean of total energy intake compared with non-carriers during pregnancy (online Supplementary Table S2). These results were confirmed, even after adjustments in the multiple linear regression model (Table 2). Women without the risk allele of FTO-rs9939609 presented negative and significant differences in the total energy intake between pregnancy and pre-pregnancy, but not women with the risk allele (online Supplementary Table S3). These results were not confirmed in the ANCOVA results (Table 3).

We observed that women with risk allele carriers of the MC4R-rs 17782313 and of the LEP-rs 7799039 genes presented lower adjusted mean of total energy intake changes from prepregnancy to pregnancy when compared with those without the risk alleles (Table 3 ).

\section{Percentage of energy from protein}

The A-allele of the FTO-rs9939609 and the C-allele of the MC4R-rs17782313 genes were associated with a decrease in the percentage of energy from protein before pregnancy and during pregnancy, respectively, in the multiple linear regression model (Table 2 ). This association was not significant for MC4R-rs17782313 based on the ANCOVA results $(P=0 \cdot 41)$ (Table 3).

Women who carried the A-allele of LEP-rs 7799039 tended to present a higher geometric mean of the percentage of energy from protein during pregnancy $(P=0.05)$ (online Supplementary Table S2) and presented a positive difference between pregnancy and pre-pregnancy (online Supplementary Table S3). The ANCOVA also indicated a significant change for percentage of energy from protein from pre-pregnancy to pregnancy, with A-allele carriers presenting a higher adjusted mean when compared with those homozygous for the G-allele $(P=0 \cdot 04)$ (Table 3).

\section{Percentage of energy from carbohydrates}

Women with the A-allele of the FTO-rs9939609 gene presented a higher mean of the percentage of energy from carbohydrates before pregnancy compared with the TT genotype (online Supplementary Table S2). These results were confirmed even after adjustment for confounders in the multiple linear regression (Table 2).

Non-carriers of risk alleles of the FTO-rs9939609 and LEPrs 7799039 genes and carriers of risk alleles of the LEPRrs1137101 and MC4R-rs17782313 genes presented positive 
Table 2. Linear regression models between gene polymorphisms and pre-pregnancy and pregnancy daily dietary intake and differences between values during pregnancy and pre-pregnancy, according to dominant inheritance models (Percentage change, linear regression coefficients $(\beta)$ and $95 \%$ confidence intervals)

\begin{tabular}{|c|c|c|c|c|c|c|c|}
\hline \multirow[b]{3}{*}{ Outcomes } & \multirow[b]{3}{*}{ Gene§ } & \multicolumn{3}{|c|}{ Pre-pregnancy* } & \multicolumn{3}{|c|}{ Pregnancy† } \\
\hline & & \multicolumn{2}{|c|}{ Adjusted $\ddagger$} & \multirow[b]{2}{*}{$P$} & \multicolumn{2}{|c|}{ Adjusted $\ddagger$} & \multirow[b]{2}{*}{$P$} \\
\hline & & $\%$ change & $95 \% \mathrm{Cl}$ & & $\%$ change & $95 \% \mathrm{Cl}$ & \\
\hline \multicolumn{8}{|c|}{ (log) total energy\|l } \\
\hline & FTO & 4.9 & $-6 \cdot 6,17 \cdot 7$ & 0.42 & $13 \cdot 3$ & $0.7,27.5$ & 0.04 \\
\hline & MC4R & -4.5 & $-14 \cdot 5,6 \cdot 6$ & 0.41 & -9.4 & $-19 \cdot 3,1 \cdot 5$ & 0.09 \\
\hline & LEP & 4.7 & $-5 \cdot 8,16 \cdot 3$ & 0.40 & $-6 \cdot 8$ & $-16 \cdot 6,4 \cdot 2$ & 0.22 \\
\hline & LEPR & $-2 \cdot 4$ & $-13 \cdot 1,9.5$ & 0.68 & $-4 \cdot 8$ & $-16 \cdot 3,8 \cdot 3$ & 0.45 \\
\hline \multicolumn{8}{|c|}{$(\log ) \%$ of energy from proteinll } \\
\hline & FTO & $-6 \cdot 5$ & $-12 \cdot 3,-0.4$ & 0.04 & $-5 \cdot 5$ & $-12 \cdot 1,1 \cdot 4$ & $0 \cdot 12$ \\
\hline & $M C 4 R$ & -2.5 & $-8.4,3.8$ & 0.43 & -7.6 & $-13 \cdot 8,-1 \cdot 0$ & 0.03 \\
\hline & LEP & -0.7 & $-6 \cdot 5,5 \cdot 3$ & 0.80 & $4 \cdot 1$ & $-2 \cdot 6,11 \cdot 2$ & 0.24 \\
\hline & LEPR & $5 \cdot 1$ & $-1 \cdot 5,12 \cdot 1$ & 0.13 & $-3 \cdot 4$ & $-10 \cdot 6,4 \cdot 3$ & 0.37 \\
\hline & & $\beta$ & $95 \% \mathrm{Cl}$ & $P$ & $\beta$ & $95 \% \mathrm{Cl}$ & $P$ \\
\hline \multicolumn{8}{|c|}{$\%$ of energy from carbohydrates } \\
\hline & FTO & $2 \cdot 6$ & $0.5,4 \cdot 8$ & 0.02 & $-0 \cdot 2$ & $-2 \cdot 0,1 \cdot 9$ & 0.98 \\
\hline & MC4R & -0.3 & $-2.4,1.9$ & 0.81 & 0.6 & $-1 \cdot 3,2 \cdot 6$ & 0.51 \\
\hline & LEP & 1.0 & $-1 \cdot 0,3 \cdot 0$ & 0.33 & -0.8 & $-2 \cdot 7,1 \cdot 2$ & 0.38 \\
\hline & LEPR & -0.5 & $-2 \cdot 7,1 \cdot 7$ & 0.67 & 1.3 & $-0 \cdot 8,3 \cdot 4$ & 0.21 \\
\hline \multicolumn{8}{|c|}{$\%$ of energy from fat } \\
\hline & FTO & $-1 \cdot 1$ & $-2 \cdot 9,0.8$ & 0.25 & 0.9 & $-0.7,2.4$ & 0.26 \\
\hline & $M C 4 R$ & 1.0 & $-0.7,2 \cdot 8$ & 0.24 & 0.6 & $-0.9,2 \cdot 1$ & 0.45 \\
\hline & LEP & -0.5 & $-2 \cdot 2,1 \cdot 2$ & 0.58 & 0.4 & $-1.5,1.6$ & 0.95 \\
\hline & $L E P R$ & -0.5 & $-2 \cdot 3,1 \cdot 4$ & 0.60 & -0.8 & $-2.4,0.9$ & 0.37 \\
\hline \multicolumn{8}{|c|}{$\%$ of energy from ultra-processed foods } \\
\hline & FTO & 1.2 & $-3 \cdot 0,5 \cdot 4$ & 0.57 & 4.5 & $-0.1,9 \cdot 0$ & 0.05 \\
\hline & MC4R & $3 \cdot 6$ & $-0.4,7 \cdot 7$ & 0.08 & 5.4 & $1 \cdot 1,9 \cdot 8$ & 0.02 \\
\hline & LEP & $1 \cdot 1$ & $-2 \cdot 9,5 \cdot 1$ & 0.58 & -0.1 & $-4 \cdot 3,4 \cdot 1$ & 0.96 \\
\hline & LEPR & -3.5 & $-7 \cdot 7,0.7$ & 0.11 & 1.6 & $-3 \cdot 2,6 \cdot 4$ & 0.51 \\
\hline
\end{tabular}

FTO, fat mass and obesity-associated gene; $M C 4 R$, melanocortin-4 receptor gene; $L E P$, leptin; $L E P R$, leptin receptor.

* Total number of observations pre-pregnancy: FTO-rs9939609, $n$ 141; MC4R-rs17782313, $n$ 140; LEP-rs7799039, $n$ 142 and LEPR-rs1137101, $n$ 142. † Total number of observations pregnancy: FTO-rs9939609, $n$ 137; MC4R-rs17782313, $n$ 136; LEP-rs7799039, $n 137$ and LEPR-rs1137101, $n 137$. $\ddagger$ All analyses were adjusted for maternal age, self-reported skin colour, parity, gestational age (week) and per-capita income; analyses of percentage of energy from macronutrients and ultra-processed foods were also adjusted for total energy intake.

$\S$ Dominant genetic model for all genes $(F T O=\mathrm{AT}+\mathrm{AA} v$. TT; $M C 4 R=\mathrm{CT}+\mathrm{CC} v$. TT; $L E P=\mathrm{GA}+\mathrm{AA} v . \mathrm{GG} ; L E P R=\mathrm{AG}+\mathrm{GG} v . \mathrm{AA})$

II Natural logarithms values were used in pre-pregnancy and pregnancy, and results were presented in $\%$ change and $95 \% \mathrm{Cl}$ to make interpretation more straightforward.

and significant differences in the percentage of energy from carbohydrates between pregnancy and pre-pregnancy (online Supplementary Table S3). These differences were not significant in the multiple linear regression models (Table 2), nor in the ANCOVA (Table 3).

\section{Percentage of energy from fat}

The percentage of energy from fat from pre-pregnancy to pregnancy reduced in the non-carriers of the risk alleles of the FTO-rs9939609 and LEP-rs 7799039 genes and in the carriers of the risk alleles of MC4R-rs17782313 and LEPR-rs1137101 genes (online Supplementary Table S3). These results were not significant in the multiple linear regression model (Table 2), nor in the ANCOVA (Table 3).

\section{Percentage of energy from ultra-processed foods}

Women who carried the A-allele of the FTO-rs9939609 gene presented a higher percentage of energy from ultra-processed foods during pregnancy when compared with those without the risk alleles (online Supplementary Table S2). These results presented borderline significance $(P=0.05)$ after adjustment for confounders in the multiple linear regression model (Table 2). The ANCOVA indicated a significant change for percentage of energy from ultra-processed foods from pre-pregnancy to pregnancy, with A-allele carriers presenting a higher adjusted mean when compared with those homozygous for the T-allele $(P=0.03)$, after adjusting for the covariate effect (energy from ultra-processed at pre-pregnancy) (Table 3).

The C-allele of MC4R-rs17782313 was positively associated with the percentage of energy from ultra-processed foods during pregnancy compared with the TT genotype (Table 2). This association was not significant based on the ANCOVA results $(P=0 \cdot 78)$ (Table 3$)$.

The percentage of energy from ultra-processed foods from pre-pregnancy to pregnancy reduced in the non-carriers of the risk alleles of the LEPR-rs1137101 gene and in the carriers of the risk alleles of MC4R-rs17782313 and of the LEP-rs 7799039 genes (online Supplementary Table S3). These results were not significant in the ANCOVA adjusted for potential confounders (Table 3). 
Table 3. Changes in dietary intake from pre-pregnancy to pregnancy, according to FTO-rs9939609, MC4R-rs17782313, LEP-rs7799039 and LEPR-rs1137101 polymorphisms ${ }^{\star}$ (Mean values and $95 \%$ confidence intervals)

\begin{tabular}{|c|c|c|c|c|c|c|}
\hline & \multicolumn{6}{|c|}{ SNP } \\
\hline & Adjusted means & \multirow[t]{3}{*}{$95 \% \mathrm{Cl}$} & Adjusted means & $95 \% \mathrm{Cl}$ & $P_{\text {interaction }} \dagger$ & $P \neq$ \\
\hline & \multirow[b]{2}{*}{ TT } & & \multicolumn{2}{|c|}{ FTO-rs9939609 (n 138) } & & \\
\hline & & & \multicolumn{2}{|c|}{$\mathrm{AT}+\mathrm{AA}$} & & \\
\hline Total energy $(\mathrm{kJ} / \mathrm{d}) \S$ & 8322 & 7071, 9795 & 9079 & 7828,10531 & 0.43 & $0 \cdot 13$ \\
\hline Total energy $(\mathrm{kcal} / \mathrm{d}) \S$ & 1989 & 1690,2341 & 2170 & 1871,2517 & 0.43 & 0.13 \\
\hline \multicolumn{7}{|l|}{$\%$ of energy from } \\
\hline Protein§ & $16 \cdot 5$ & $14 \cdot 8,18 \cdot 3$ & $15 \cdot 8$ & $14 \cdot 4,17 \cdot 4$ & 0.37 & 0.30 \\
\hline Carbohydrates & $59 \cdot 0$ & $56 \cdot 1,61 \cdot 8$ & 58.5 & $55 \cdot 9,61 \cdot 2$ & 0.59 & 0.68 \\
\hline Fat & $23 \cdot 3$ & $21 \cdot 0,25 \cdot 6$ & 24.5 & $22 \cdot 4,26 \cdot 6$ & 0.31 & $0 \cdot 16$ \\
\hline \multirow[t]{2}{*}{ Ultra-processed foods } & 39.1 & $32 \cdot 5,45 \cdot 7$ & 44.2 & $38 \cdot 3,50 \cdot 2$ & 0.95 & 0.03 \\
\hline & \multicolumn{2}{|c|}{ TT } & \multicolumn{2}{|c|}{ MC4R-rs17782313 (n 137) } & & \\
\hline Total energy $(\mathrm{kJ} / \mathrm{d}) \S$ & 9155 & 7904,10602 & 7966 & 6774,9372 & 0.64 & 0.02 \\
\hline Total energy $(\mathrm{kcal} / \mathrm{d}) \S$ & 2188 & 1889,2534 & 1904 & 1619,2240 & 0.64 & 0.02 \\
\hline \multicolumn{7}{|l|}{$\%$ of energy from } \\
\hline Protein§ & $16 \cdot 1$ & $14 \cdot 6,17 \cdot 7$ & $15 \cdot 6$ & $14 \cdot 0,17 \cdot 3$ & 0.94 & 0.41 \\
\hline Carbohydrates & $58 \cdot 1$ & $55 \cdot 5,60 \cdot 6$ & 59.5 & $56 \cdot 6,62 \cdot 3$ & 0.78 & 0.18 \\
\hline Fat & $24 \cdot 6$ & $22 \cdot 4,26 \cdot 7$ & 23.8 & $21 \cdot 4,26 \cdot 2$ & 0.75 & 0.36 \\
\hline \multirow[t]{3}{*}{ Ultra-processed foods } & $42 \cdot 4$ & $36 \cdot 3,48 \cdot 5$ & $43 \cdot 1$ & $36 \cdot 4,49 \cdot 8$ & 0.23 & 0.78 \\
\hline & \multirow{2}{*}{\multicolumn{2}{|c|}{ GG }} & \multicolumn{2}{|c|}{ LEP-rs7799039 (n 138) } & & \\
\hline & & & $\mathrm{GA}$ & & & \\
\hline Total energy $(\mathrm{kJ} / \mathrm{d}) \S$ & 9171 & 7908,10636 & 8217 & 7046, 9581 & 0.28 & 0.04 \\
\hline Total energy $(\mathrm{kcal} / \mathrm{d}) \S$ & 2192 & 1890,2542 & 1964 & 1684,2290 & 0.28 & 0.04 \\
\hline \multicolumn{7}{|l|}{$\%$ of energy from } \\
\hline Protein§ & $15 \cdot 6$ & $14 \cdot 2,17 \cdot 2$ & $16 \cdot 8$ & $15 \cdot 2,18 \cdot 5$ & 0.20 & 0.04 \\
\hline Carbohydrates & $59 \cdot 0$ & $56 \cdot 4,61 \cdot 7$ & 57.9 & $55 \cdot 1,60 \cdot 6$ & 0.07 & 0.22 \\
\hline Fat & $24 \cdot 3$ & $22 \cdot 1,26 \cdot 6$ & $24 \cdot 1$ & $21 \cdot 7,26 \cdot 5$ & 0.97 & 0.78 \\
\hline \multirow{2}{*}{ Ultra-processed foods } & 43.9 & $37 \cdot 2,49 \cdot 5$ & $41 \cdot 1$ & $34.8,47.5$ & 0.58 & 0.30 \\
\hline & \multicolumn{2}{|c|}{ AA } & \multicolumn{2}{|c|}{$\begin{array}{l}\text { LEPR-rs1137101 (n 138) } \\
\quad \mathrm{AG}+\mathrm{GG}\end{array}$} & & \\
\hline Total energy $(\mathrm{kJ} / \mathrm{d}) \S$ & 9180 & 7732,10895 & 8569 & 7422,9891 & 0.96 & 0.26 \\
\hline Total energy $(\mathrm{kcal} / \mathrm{d}) \S$ & 2194 & 1848,2604 & 2048 & 1774,2364 & 0.96 & 0.26 \\
\hline \multicolumn{7}{|l|}{$\%$ of energy from } \\
\hline Protein§ & $16 \cdot 5$ & $14 \cdot 8,18 \cdot 4$ & $15 \cdot 8$ & $14 \cdot 4,17 \cdot 4$ & 0.45 & 0.32 \\
\hline Carbohydrates & 57.3 & $54 \cdot 2,60 \cdot 3$ & $58 \cdot 7$ & $56 \cdot 2,61 \cdot 2$ & 0.48 & 0.17 \\
\hline Fat & $25 \cdot 1$ & $22 \cdot 6,27 \cdot 6$ & $24 \cdot 2$ & $22 \cdot 1,26 \cdot 2$ & 0.15 & 0.30 \\
\hline Ultra-processed foods & 41.0 & $33 \cdot 9,48 \cdot 2$ & $42 \cdot 8$ & $36 \cdot 8,48 \cdot 8$ & 0.26 & 0.49 \\
\hline
\end{tabular}

FTO, fat mass and obesity-associated gene; MC4R, melanocortin-4 receptor gene; LEP, leptin; LEPR, leptin receptor.

* All analyses were adjusted for pre-pregnancy dietary intake (continuous variables), maternal age, self-reported skin colour, parity and gestational age (week) at baseline.

$\dagger P$ value for interaction SNP $\times$ pre-pregnancy dietary intake.

$\ddagger P$ value obtained from ANCOVA for the effect of $F T O, M C 4 R, L E P$ and $L E P R$ polymorphisms on the change of each variable.

$\S$ Natural logarithms values were used for analyses, but estimates provided in the table were back-transformed to make interpretation more straightforward.

\section{Discussion}

This study has several main findings. The A-allele of FTOrs9939609 was associated with a decrease in the percentage of energy from protein and positively associated with the percentage of energy from carbohydrates before pregnancy and positively associated with change in the percentage of energy from ultra-processed foods from pre-pregnancy to pregnancy. C-allele of MC4R-rs17782313 was associated with change in the total energy intake from pre-pregnancy to pregnancy, with C-allele carriers presenting a lower adjusted mean when compared with non-carriers. A-allele of LEP-rs 7799039 was associated with dietary intake change from pre-pregnancy to pregnancy, with carriers presenting lower total energy intake adjusted mean and higher adjusted mean in the percentage of energy from protein when compared with non-carriers.

The minor frequency alleles (MAF) observed in this study were quite similar to the range of reported values in other published studies with a Brazilian population for FTOrs9939609 (0.40/0.45) and MC4R-rs17782313 $(0 \cdot 15 / 0 \cdot 23)^{(31)}$ and for LEP-rs $7799039(0 \cdot 27 / 0 \cdot 30)^{(32)}$. The MAF for $L E P R$ rs1137101 in this study was higher than that reported in a study of multi-ethnic subjects in the same city as our study (0.39/ $0.43)^{(32)}$ but similar to 0.45 - the mean rate (ranged from 0.32 to 0.58 ) that was observed in different Caucasian populations and in those of Western European descent $(0 \cdot 47)^{(33,34)}$. In this study, the MAF of the A-allele at the rs9939609 variant is also comparable to that in Pakistani $(0 \cdot 40)$ and Italian $(0.43)$ studies ${ }^{(35,36)}$ but higher than that reported in a sample of the Chinese population $(0 \cdot 12)^{(37)}$. The MAF of the C-allele at the $r s 17782313$ variant is lower than that reported in Italian (0.28) and Korean $(0 \cdot 26)$ populations $^{(36,38)}$.

Recent gene identification efforts have provided a more comprehensive picture of the biological mechanisms involved in the development of obesity. Many of the obesity-susceptibility genes are highly expressed in the hypothalamus, a crucial neural centre 
for energy balance and regulation of food intake ${ }^{(39)}$. Overweight and obesity are the results of a complex interplay between genetic determinants and conditions associated with lifestyle and the environment that have additive and interactive effects. It is worth noting that this is the first study, to the best of our knowledge, that has examined the association between FTO-rs9939609, MC4Rrs17782313, LEP-rs 7799039 and LEPR-rs1137101 polymorphisms and dietary intake during pregnancy, proving it difficult to compare our data.

The physiologic pathway by which variation in the FTO gene influences the risk of developing obesity largely remains to be established. A study showed that subjects homozygous for FTOrs9939609 appear to determine neural responses to circulating concentrations of the hunger hormone ghrelin ${ }^{(40)}$, which may lead to increased energy intake in those carrying the risk allele. The role of FTO in the pathogenesis of obesity has additionally been demonstrated in a rodent model, which indicated that increased expression of FTO leads to increased fat mass and obesity via hyperphagia ${ }^{(41)}$

Research involving humans has suggested that the risk allele of the FTO-rs9939609 gene may influence food consumption parameters including total energy intake ${ }^{(42,43)}$, food preferences $^{(44,42)}$ and appetite regulation ${ }^{(7,33)}$, suggesting that diet may mediate the effect of FTO on obesity. However, these associations have not been replicated in other studies ${ }^{(45,46)}$. We observed that this variant was associated with a decrease in the percentage of energy from protein and positively associated with the percentage of energy from carbohydrates before pregnancy and also associated with change in the percentage of energy from ultra-processed foods from pre-pregnancy to pregnancy. Protein is considered the most satiating macronutrient ${ }^{(47)}$; thus, it is plausible that the A-allele of FTO was related to modifications of food cravings and appetite with increased energy intake from carbohydrates and ultraprocessed foods.

The MC4R gene is the most common cause of severe early monogenic obesity ${ }^{(48)}$ and an important contributor to polygenic obesity in humans ${ }^{(12)}$. However, its expression is variable, its penetrance is incomplete and both its expression and penetrance are age-dependent ${ }^{(49)}$. In addition, the incomplete penetrance and the variable expression of $M C 4 R$ mutations suggest an important environmental influence. The phenotypic features of $M C 4 R$ deficiency include hyperphagia ${ }^{(12,48)}$ through the leptin-melanocortin signalling system ${ }^{(50)}$; the severity of hyperphagia may decline with age and it was greater in homozygous compared with heterozygous cases ${ }^{(48,49)}$. In our study, the majority of the pregnant women were heterozygous, with only four homozygous subjects with the minor allele. Therefore, we were unable to test this possibility.

The MC4R-rs17782313 C-allele has been associated with high snack consumption ${ }^{(11)}$ and with high intakes of total energy and dietary fat in women of European ancestry ${ }^{(51)}$. We observed that MC4R-rs17782313 genotypes were not associated with daily energy intake before pregnancy. This is consistent with the results of studies in Dutch ${ }^{(13)}$ and Danish ${ }^{(46)}$ women. We also found that the MC4R-rs17782313 gene was significantly associated with changes in total energy intake from pre-pregnancy to pregnancy, independently of pre-pregnancy energy intake. However, C-allele carriers did not present higher energy intake adjusted means.

In the current study, the association of the MC4R-rs 17782313 and the percentage of energy from ultra-processed foods from pre-pregnancy to pregnancy lost significance when the results were adjusted for pre-pregnancy dietary intake. In contrast, a study in the Korean population reported that the $M C 4 R$ rs17782313 C-allele displayed a positive association with ramen (Japanese dish consisting of wheat noodle mixed with flavoured broth and a number of different toppings) and processed foods including canned tuna, fish cake, ham and cheese ${ }^{(38)}$.

There are limited studies evaluating the association of $L E P$ rs 7799039 and its receptor (LEPR-rs1137101) with food intake in the adult population. The LEPR-rs1137101 SNP resulting in amino acid substitution Gln223Arg was earlier hypothesised to affect the functionality of the $L E P R$, with the $G$ allele resulting in reduced $L E P$ sensitivity ${ }^{(52)}$. Resistance to leptin is known to increase food intake. Research conducted in Brazil on children (4 years of age) found that G-allele carriers of LEPR-rs1137101, but not LEP-rs 7799039, had a higher total energy intake ${ }^{(53)}$. In Tunisian adults, subjects with the AA genotype for $L E P$ rs 7799039 and GG genotype for LEPR-rs1137101 genes had a significantly higher daily energy intake ${ }^{(10)}$. We did not find an association between LEPR-rs1137101 SNP and dietary intake in pregnant women, but found that A-allele of LEP-rs 7799039 was associated with dietary intake change from pre-pregnancy to pregnancy, with carriers presenting lower total energy intake adjusted mean and higher adjusted mean of the percentage of energy from protein when compared with non-carriers.

There is evidence to conclude that obesity candidate gene SNP may be associated with dietary intake parameters, and their identification may allow the development of tailored interventions to prevent future health problems. However, phenotype intensity is potentially modulated by environmental and individual char$\operatorname{acteristics}^{(12)}$, and the genetic contribution to population phenotypic differentiation is driven by differences in causal allele frequencies, effect sizes and genetic architecture ${ }^{(54)}$.

Our study presents limitations and strengths that need to be highlighted. One of the limitations of the present study is the limited sample size and lack of formal power calculation, as this study was designed to be exploratory and hypothesis-generating in nature and not confirmatory. Therefore, the results should be taken with caution because our study is underpowered and other studied associations could have been missed in the statistical analysis. Corrections for multiple testing were not performed in the present study. The statistical significant results could therefore include false positives. However, it provides important information that may be useful in future studies to consolidate whether genes susceptible to obesity are associated with dietary intake in pregnant women, which should be confirmed in larger cohorts of pregnant women. An additional limitation is that the FFQ previously validated for adults ${ }^{(22)}$ was not developed specifically for pregnancy, but represent the usual dietary intake of the population who lives in Rio de Janeiro. Epidemiological studies have shown that an FFQ can be used to measure nutritional dietary intake during pregnancy with acceptable reproducibility and represents a useful tool for categorising pregnant women according to their dietary intake ${ }^{(5)}$, including in Brazil ${ }^{(56)}$. 
Moreover, there was no validated FFQ for pregnant women in Brazil when this cohort study began.

In conclusion, the A-allele carriers of FTO-rs9939609 were positively associated with the percentage of energy from carbohydrates and with a decrease in the percentage of energy from protein before pregnancy and with change in the percentage of energy from ultra-processed foods from pre-pregnancy to pregnancy compared with those with the TT-genotype. The C-allele of MC4R-rs17782313 was associated with change in the total energy intake from pre-pregnancy to pregnancy, with C-allele carriers presenting a lower adjusted mean than noncarriers. There was a significant association between A-allele of LEP-rs 7799039 and change in dietary intake from prepregnancy to pregnancy, with carriers presenting lower total energy intake adjusted mean and higher adjusted mean of percentage of energy from protein when compared with noncarriers, but there was no association of LEPR-rs1137101 with total energy intake and percentage of energy from macronutrients or ultra-processed foods.

Genes might interact with each other or with environmental factors such as food nutrients to play a role in the development of adiposity. Further research using high-quality dietary data is required to consolidate whether obesity-susceptible genes are associated with dietary intake to allow the application of tailored interventions to prevent obesity in genetically predisposed individuals

\section{Acknowledgements}

The authors would like to acknowledge Professor Rosane Silva for her technical support in the genotyping analysis and for allowing us to conduct DNA extraction in the Laboratory of Macromolecular Metabolism Firmino Torres de Castro, Biophysics Institute, Rio de Janeiro Federal University (UFRJ). The authors also thank Professor Maria das Graças Tavares do Carmo for allowing us to work at the Laboratory of Nutritional Biochemistry of the Nutrition Institute, UFRJ.

This study was supported by the Carlos Chagas Filho Research Foundation from the State of Rio de Janeiro (FAPERJ) (grant nos E-26/111.400/2010, E-26/110·681/2012; E-26/ $112 \cdot 181 / 2012$; E-26/111·698/2013). The funders had no role in the design, analysis or writing of this article.

The authors' contributions are as follows: M. C. M. performed statistical analysis, and participated in the interpretation of results and writing of the manuscript. J. T. participated in statistical analysis and contributed to the discussion and data interpretation. A. A. F.-V. and D. R. F. participated in the data collection and contributed to the discussion and data interpretation. E. L. R. and C. J. S. contributed to the discussion and data interpretation. G. K. participated in the designing the work, contributed to the discussion and data interpretation and reviewed the manuscript.

The authors declare that there are no conflicts of interest.

\section{Supplementary material}

For supplementary material/s referred to in this article, please visit https://doi.org/10.1017/S0007114518001423

\section{References}

1. Malik VS, Willett WC \& Hu FB (2013) Global obesity: trends, risk factors and policy implications. Nat Rev Endocrinol 9, 13-27.

2. Ng M, Fleming T, Robinson M, et al. (2014) Global, regional, and national prevalence of overweight and obesity in children and adults during 1980-2013: a systematic analysis for the Global Burden of Disease Study 2013. Lancet 384, 766-781.

3. Imamura F, Micha R, Khatibzadeh S, et al. (2015) Dietary quality among men and women in 187 countries in 1990 and 2010: a systematic assessment. Lancet Glob Health 3, e132-e142.

4. Herrera BM \& Lindgren CM (2010) The genetics of obesity. Curr Diab Rep 10, 498-505.

5. Doo M \& Kim Y (2015) Obesity: interactions of genome and nutrients intake. Prev Nutr Food Sci 20, 1-7.

6. Tanaka T (2014) Genetics of energy and macronutrient intake in humans. Curr Nutr Rep 3, 170-177.

7. Dougkas A, Yaqoob P, Givens DI, et al. (2013) The impact of obesity-related SNP on appetite and energy intake. Br J Nutr 110, 1151-1156.

8. Mariman EC, Bouwman FG, Aller EE, et al. (2015) Extreme obesity is associated with variation in genes related to the circadian rhythm of food intake and hypothalamic signaling. Physiol Genomics 47, 225-231.

9. Sonestedt E, Roos C, Gullberg B, et al. (2009) Fat and carbohydrate intake modify the association between genetic variation in the FTO genotype and obesity. Am J Clin Nutr 90, $1418-1425$

10. Boumaiza I, Omezzine A, Rejeb J, et al. (2012) Relationship between leptin G2548A and leptin receptor Q223R gene polymorphisms and obesity and metabolic syndrome risk in Tunisian volunteers. Genet Test Mol Biomarkers 16, 726-733.

11. Stutzmann F, Cauchi S, Durand E, et al. (2009) Common genetic variation near MC4R is associated with eating behaviour patterns in European populations. Int J Obes (Lond) $\mathbf{3 3}$, 373-378.

12. Cecil J, Dalton M, Finlayson G, et al. (2012) Obesity and eating behaviour in children and adolescents: contribution of common gene polymorphisms. Int Rev Psychiatry 24, 200-210.

13. Bauer F, Elbers CC, Adan RA, et al. (2009) Obesity genes identified in genome-wide association studies are associated with adiposity measures and potentially with nutrient-specific food preference. Am J Clin Nutr 90, 951-959.

14. Pan American Health Organization (2015) Ultra-Processed Food and Drink Products in Latin America: Trends, Impact on Obesity, Policy Implications. Washington, DC: Pan American Health Organization.

15. Martínez Steele E, Popkin BM, Swinburn B, et al. (2017) The share of ultra-processed foods and the overall nutritional quality of diets in the US: evidence from a nationally representative cross-sectional study. Popul Health Metr 15, 6.

16. Ministry of Health of Brazil (2015) Dietary Guidelines for the Brazilian Population, 2nd ed. Brasilia: Ministry of Health of Brazil.

17. Monteiro CA, Cannon G, Levy R, et al. (2016) NOVA. The star shines bright. [Food classification. Public health]. World Nutr 7, 28-38.

18. Plećaš D, Plešinac S \& Kontić-Vučinić O (2014) Nutrition in pregnancy: basic principles and recommendations. Srp Arb Celok Lek 142, 125-130.

19. Ovesen P, Rasmussen S \& Kesmodel U (2011) Effect of prepregnancy maternal overweight and obesity on pregnancy outcome. Obstet Gynecol 118, 305-312.

20. Marchi J, Berg M, Dencker A, et al. (2015) Risks associated with obesity in pregnancy, for the mother and baby: a systematic review of reviews. Obes Rev 16, 621-638. 
21. Morgan KL, Rahman MA, Macey S, et al. (2014) Obesity in pregnancy: a retrospective prevalence-based study on health service utilisation and costs on the NHS. BMJ Open $\mathbf{4}$ e003983.

22. Sichieri R \& Everhart JE (1998) Validity of a Brazilian food frequency questionnaire against dietary recalls and estimated energy intake. Nutr Res 18, 1649-1659.

23. Pinheiro ABV, Lacerda EM, de A, Benzecry EH, et al. (2004) Tabela para avaliação de consumo alimentar em medidas caseiras, 5th ed. São Paulo: Atheneu.

24. Núcleo de Estudos e Pesquisas em Alimentação (NEPA), Universidade Estadual de Campinas (UNICAMP) (2011) Tabela Brasileira de Composição de Alimentos (TACO), 4th ed. Campinas-SP: NEPA/UNICAMP.

25. US Department of Agriculture (2011) National nutrient database for standard reference, release 24. http://www.ars.usda. gov/ba/bhnrc/ndl (accessed March 2017).

26. Lohman TG, Roche AF \& Martorell R (1988) Antbropometric Standardization Reference Manual. Champaign, IL: Human Kinetics Books.

27. Butt K, Lim K, Bly S, et al. (2014) Determination of gestational age by ultrasound. J Obstet Gynaecol Can 36, 171-181.

28. R Core Team (2017) R: A Language and Environment for Statistical Computing. Vienna, Austria: R Foundation for Statistical Computing.

29. Faul F, Erdfelder E, Buchner A, et al. (2009) Statistical power analyses using $G^{*}$ Power 3.1: tests for correlation and regression analyses. Behav Res Methods 41, 1149-1160.

30. Perneger TV (1998) What's wrong with Bonferroni adjustments. BMJ 316, 1236-1238.

31. Da Cunha PA, de Carlos Back LK, Sereia AFR, et al. (2013) Interaction between obesity-related genes, FTO and MC4R, associated to an increase of breast cancer risk. Mol Biol Rep 40, 6657-6664.

32. Duarte S, Francischetti E, Genelhu V, et al. (2006) LEPR p. Q223R, beta3-AR p. W64R and LEP c.-2548G> A gene variants in obese Brazilian subjects. Genet Mol Res 6, $1035-1043$.

33. den Hoed M, Westerterp-Plantenga MS, Bouwman FG, et al. (2009) Postprandial responses in hunger and satiety are associated with the rs9939609 single nucleotide polymorphism in FTO. Am J Clin Nutr 90, 1426-1432.

34. Paracchini V, Pedotti P \& Taioli E (2005) Genetics of leptin and obesity: a HuGE review. Am J Epidemiol 162, 101-114.

35. Fawwad A, Siddiqui IA, Basit A, et al. (2016) Common variant within the FTO gene, rs9939609, obesity and type 2 diabetes in population of Karachi, Pakistan. Diabetes Metab Syndr Clin Res Rev 10, 43-47.

36. Bordoni L, Marchegiani F, Piangerelli M, et al. (2017) Obesity-related genetic polymorphisms and adiposity indices in a young Italian population. IUBMB Life 69, 98-105.

37. Li H, Wu Y, Loos RJ, et al. (2008) Variants in the fat mass-and obesity-associated (FTO) gene are not associated with obesity in a Chinese Han population. Diabetes 57, 264-268.

38. Park S, Daily JW, Zhang X, et al. (2016) Interactions with the MC4R rs17782313 variant, mental stress and energy intake and the risk of obesity in genome epidemiology study. Nutr Metab (Lond) 13, 38.

39. Hofker M \& Wijmenga C (2009) A supersized list of obesity genes. Nat Genet 41, 139-140.
40. Karra E, O'Daly OG, Choudhury AI, et al. (2013) A link between FTO, ghrelin, and impaired brain food-cue responsivity. J Clin Invest 123, 3539-3551.

41. Church C, Moir L, McMurray F, et al. (2010) Overexpression of Fto leads to increased food intake and results in obesity. Nat Genet 42, 1086-1092.

42. Cecil JE, Tavendale R, Watt P, et al. (2008) An obesityassociated FTO gene variant and increased energy intake in children. $N$ Engl J Med 359, 2558-2566.

43. Qi Q, Downer MK, Kilpeläinen TO, et al. (2015) Dietary intake, FTO genetic variants and adiposity: a combined analysis of over 16,000 children and adolescents. Diabetes $\mathbf{6 4}$, $2467-2476$

44. Brunkwall L, Ericson U, Hellstrand S, et al. (2013) Genetic variation in the fat mass and obesity-associated gene (FTO) in association with food preferences in healthy adults. Food Nutr Res 57, 20028

45. Liu G, Zhu H, Lagou V, et al. (2010) FTO variant rs9939609 is associated with body mass index and waist circumference, but not with energy intake or physical activity in European-and African-American youth. BMC Med Genet 11, 57.

46. Hasselbalch AL, Ängquist L, Christiansen L, et al. (2010) A variant in the fat mass and obesity-associated gene (FTO) and variants near the melanocortin- 4 receptor gene (MC4R) do not influence dietary intake. J Nutr 140, 831-834.

47. Morell P \& Fiszman S (2017) Revisiting the role of proteininduced satiation and satiety. Food Hydrocoll 68, 199-210.

48. Farooqi IS, Keogh JM, Yeo GS, et al. (2003) Clinical spectrum of obesity and mutations in the melanocortin 4 receptor gene. $N$ Engl J Med 348, 1085-1095.

49. Stutzmann F, Tan K, Vatin V, et al. (2008) Prevalence of melanocortin- 4 receptor deficiency in Europeans and their age-dependent penetrance in multigenerational pedigrees. Diabetes 57, 2511-2518.

50. Girardet C \& Butler AA (2014) Neural melanocortin receptors in obesity and related metabolic disorders. Biochim Biophys Acta 1842, 482-494.

51. Qi L, Kraft P, Hunter DJ, et al. (2008) The common obesity variant near MC4R gene is associated with higher intakes of total energy and dietary fat, weight change and diabetes risk in women. Hum Mol Genet 17, 3502-3508.

52. Yiannakouris N, Yannakoulia M, Melistas L, et al. (2001) The Q223R polymorphism of the leptin receptor gene is significantly associated with obesity and predicts a small percentage of body weight and body composition variability. J Clin Endocrinol Metab 86, 4434-4439.

53. Zandoná MR, Rodrigues RO, Albiero G, et al. (2013) Polymorphisms in LEPR, PPARG and APM1 genes: associations with energy intake and metabolic traits in young children. Arq Bras Endocrinol Metabol 57, 603-611.

54. Brown BC, Ye CJ, Price AL, et al. (2016) Transethnic geneticcorrelation estimates from summary statistics. Am J Hum Genet 99, 76-88.

55. Vioque J, Navarrete-Muñoz E-M, Gimenez-Monzó D, et al. (2013) Reproducibility and validity of a food frequency questionnaire among pregnant women in a Mediterranean area. Nutr J 12, 26.

56. Barbieri P, Nishimura RY, Crivellenti LC, et al. (2013) Relative validation of a quantitative FFQ for use in Brazilian pregnant women. Public Health Nutr 16, 1419-1426. 